\title{
Identification of $\mathrm{BCL}-\mathrm{XL}$ as highly active survival factor and promising therapeutic target in colorectal cancer
}

\author{
Anna-Lena Scherr ${ }^{1}$, Andreas Mock ${ }^{1,2,3}$, Georg Gdynia ${ }^{4}$, Nathalie Schmitt ${ }^{1}$, Christoph E. Heilig9 ${ }^{2,3}$, Felix Korell, \\ Praveen Rhadakrishnan ${ }^{6}$, Paula Hoffmeister', Klaus H. Metzeler ${ }^{7}$, Klaus Schulze-Osthoff ${ }^{3,8}$, Anna L. Illert 9,10,11, \\ Melanie Boerries ${ }^{10,11,12}$, Jörg Trojan ${ }^{13,14}$, Oliver Waidmann ${ }^{13,14}$, Johanna Falkenhorst ${ }^{15,16}$, Jens Siveke ${ }^{17,18}$, \\ Philipp J. Jost $\mathbb{B}^{19,20,21}$, Michael Bitzer22, Nisar P. Malek ${ }^{22}$, Loredana Vecchione ${ }^{23,24}$, Ivan Jelas ${ }^{23}$, Benedikt Brors ${ }^{3,25}$, \\ Hanno Glimm 26,27,28, Albrecht Stenzinger 3,4, Svetlana P. Grekova ${ }^{4}$, Tobias Gehrig ${ }^{29}$, Henning Schulze-Bergkamen ${ }^{30}$, \\ Dirk Jäger ${ }^{1,3}$, Peter Schirmacher ${ }^{3,4}$, Mathias Heikenwalder ${ }^{31}$, Benjamin Goeppert ${ }^{4}$, Martin Schneider ${ }^{6}$, \\ Stefan Fröhling $\mathbb{1 D}^{2,3}$ and Bruno C. Köhler ${ }^{1,3}$
}

\begin{abstract}
Since metastatic colorectal cancer (CRC) is a leading cause of cancer-related death, therapeutic approaches overcoming primary and acquired therapy resistance are an urgent medical need. In this study, the efficacy and toxicity of high-affinity inhibitors targeting antiapoptotic $B C L-2$ proteins $(B C L-2, B C L-X L$, and $M C L-1)$ were evaluated. By RNA sequencing analysis of a pan-cancer cohort comprising $>1500$ patients and subsequent prediction of protein activity, BCL-XL was identified as the only antiapoptotic BCL-2 protein that is overactivated in CRC. Consistently, pharmacologic and genetic inhibition of BCL-XL induced apoptosis in human CRC cell lines. In a combined treatment approach, targeting $B C L-X L$ augmented the efficacy of chemotherapy in vitro, in a murine CRC model, and in human ex vivo derived CRC tissue cultures. Collectively, these data show that targeting of $B C L-X L$ is efficient and safe in preclinical CRC models, observations that pave the way for clinical translation.
\end{abstract}

\section{Introduction}

Colorectal cancer (CRC) ranks among the most frequent cancers worldwide and represents a leading cause of cancer-related death ${ }^{1,2}$. In the metastasized situation (UICC stage IV), chemotherapy remains the backbone of standard of care therapeutic approaches ${ }^{3}$. In addition to poly-chemotherapy, targeting EGFR and VEGFR complements therapeutic regimes in clinical practice ${ }^{4}$. In the

\footnotetext{
Correspondence: Bruno C. Köhler (bruno.koehler@nct-heidelberg.de)

'Department of Medical Oncology, National Center for Tumor Diseases (NCT), University Hospital Heidelberg, 69120 Heidelberg, Germany

${ }^{2}$ Department of Translational Medical Oncology, National Center for Tumor Diseases (NCT) Heidelberg, German Cancer Research Center (DKFZ), 69120 Heidelberg, Germany

Full list of author information is available at the end of the article

Edited by K. Sarosiek
}

attempt to reach a more personalized patient stratification, the mutational landscape including pan-RAS, BRAF, MSI-status as well as sidedness of the tumor is taken into account ${ }^{3,5-8}$. Even if systemic treatment of metastatic CRC underwent fundamental improvements, curation is not in sight. Thus, an evident clinical need to improve CRC treatment prompted us to study novel combinatorial approaches.

Primary treatment failure as well as acquired resistance is determined by defective cell death signaling ${ }^{9-11}$. In several studies it has been shown that cells accumulate proapoptotic proteins in the course of oncogenic transformation, caused by check point evasion and DNA damage response ${ }^{12,13}$. Nevertheless, a considerable percentage of tumor cells manages to escape from cell death

\section{(c) The Author(s) 2020}

(c) (i) Open Access This article is licensed under a Creative Commons Attribution 4.0 International License, which permits use, sharing, adaptation, distribution and reproduction c. in any medium or format, as long as you give appropriate credit to the original author(s) and the source, provide a link to the Creative Commons license, and indicate if changes were made. The images or other third party material in this article are included in the article's Creative Commons license, unless indicated otherwise in a credit line to the material. If material is not included in the article's Creative Commons license and your intended use is not permitted by statutory regulation or exceeds the permitted use, you will need to obtain permission directly from the copyright holder. To view a copy of this license, visit http://creativecommons.org/licenses/by/4.0/. 
induction by counterbalancing increased levels of proapoptotic proteins with upregulation of antiapoptotic factors $^{14-16}$. In this context, the BCL-2 family of proteins plays an essential role ${ }^{17}$. The family comprises pro and antiapoptotic proteins opposing each other. Among antiapoptotic BCL-2 proteins, BCL-2 itself, BCL-XL and MCL-1 are most prominent. They sequester and thereby inhibit the proapoptotic multidomain BCL-2 proteins, BAX and BAK, which assemble to pores in the outer mitochondrial membrane to initiate the intrinsic pathway of apoptotic cell death ${ }^{18}$. A third group of proteins, the BH3-only proteins, contribute to apoptosis either by competing with BAX or BAK for the binding of antiapoptotic proteins or by directly activating them for cytochrome c release.

The vision of utilizing this delicate balance to drive tumor cells over the edge of apoptosis induction, led to the development of small-molecule inhibitors targeting antiapoptotic protein function (BH3-mimetics) ${ }^{19}$. The BCL-2 specific inhibitor venetoclax (ABT-199) was the first compound in this field that became approved by the United States Food and Drug Administration (FDA) for treatment of chronic lymphocytic leukemia (CLL) and selected acute myeloid leukemia (AML) patients ${ }^{20-23}$.

In contrast to hematologic malignancies, where BCL-2 seems most important for tumor cell survival, the response of solid tumors to antiapoptotic BCL-2 protein inhibition is far more complex, due to their heterogeneity. Amplification of the genes coding for MCL-1 and BCL$\mathrm{XL}$ (BCL2L1) has been found to rank among the most common copy number alterations in various solid tumor entities $^{24}$. In the context of CRC, especially BCL-XL has been identified as a driver of oncogenesis and tumor progression $^{25,26}$. In a murine CRC model, we have previously shown that specific deletion of $\mathrm{BCL}-\mathrm{XL}$ in the intestinal epithelium renders mice less susceptible towards chemically induced carcinogenesis via increasing apoptosis induction in tumor cells $\mathrm{s}^{25}$.

Following our preceding studies, and equipped with new and highly specific BH3-mimetics, the aim of this study was to further delineate the potential of targeting antiapoptotic BCL- 2 proteins, especially $\mathrm{BCL}-\mathrm{XL}$, in the context of CRC treatment in terms of efficacy, toxicity and as a combinatorial approach with standard-of care therapeutic agents.

\section{Methods and materials}

\section{RNA sequencing and estimation of protein activity in the} NCT/DKTK MASTER cohort

The MASTER (Molecularly Aided Stratification for Tumor Eradication Research) program of the NCT Heidelberg and the German Cancer Consortium (DKTK) is a registry trial and analytical platform for prospective, omics-driven stratification of younger adults with advanced-stage cancer across all histologies and patients with rare tumors ${ }^{27}$. As of September 2019, a total of 1521 patient samples, including 68 from patients with metastatic CRC, have been analyzed by RNA sequencing. Furthermore, the activity of 6014 proteins was inferred from RNA sequencing data by Master Regulator Analy$\mathrm{sis}^{28}$ employing the metaVIPER algorithm ${ }^{29}$. The viper $\mathrm{R}$ package $^{30}$ was used to estimate the protein activity in an entity-independent fashion by integrating regulatory networks from 24 different TCGA tumor entities. The regulatory networks were reverse engineered by the ARACNe (algorithm for the reconstruction of accurate cellular networks) algorithm ${ }^{31}$ and are available through the aracne.network $\mathrm{R}$ package ${ }^{32}$. Through the integration of interactomes, the activity of 6013 proteins that were adequately represented in at least one of the available regulatory networks could be inferred in the MASTER cohort.

\section{Cell lines and reagents}

The human CRC cell lines Colo205, HT29, CaCo2 and SW480 as well as the nontransformed human colon cell line CCD 841 CoN were purchased from ATCC (Manassas Virginia, USA). CRC cells were maintained in RPMI + GlutaMAX medium (Gibco, Karlsruhe, Germany) and CCD $841 \mathrm{CoN}$ cells in DMEM medium (Gibco), in each case supplemented with $10 \%$ heat-inactivated fetal calf serum (PAA Laboratories, Cölbe, Germany) and 1\% penicillin/streptomycin (PAA Laboratories) and cultured in a humidified atmosphere at $37^{\circ} \mathrm{C}$ and $5 \% \mathrm{CO}_{2}$. Staurosporine and chemotherapeutic reagents 5 -fluorouracil $(5 \mathrm{FU})$ and irinotecan were purchased from Sigma-Aldrich (Munich, Germany). Selective inhibitors ABT-199 (BCL-2 inhibitor), S63845 (MCL-1 inhibitor), and A-1331852 (orally available BCL-XL inhibitor, used for murine experiments) were purchased from Selleckchem (Munich, Germany) and WEHI-539 (BCL-XL inhibitor, used for in vitro and ex vivo experiments) was purchased from Hycultec (Beutelsbach, Deutschland).

\section{Protein isolation, SDS-PAGE and western blot analysis}

For protein analysis, cells were seeded and treated as indicated. Protein isolation was performed as described previously $^{33}$ and equal amounts of protein were separated by $12 \%$ SDS-PAGE and blotted onto nitrocellulose membranes by standard procedures. Immunoblotting was performed using the following primary antibodies: BCLXL (\# 2764, Cell Signaling Technology, Danvers, MA, USA), MCL-1 (\# sc-819, Santa Cruz Biotechnology, Heidelberg, Germany), BCL-2 (\# ab692, Abcam, Cambridge, UK), and Tubulin (\# T8203, Sigma-Aldrich) as well as peroxidase-conjugated secondary antibodies (Santa Cruz Biotechnology). Bound antibody was visualized using an enhanced chemiluminescence detection system (Perkin 
Elmer, Zaventem, Belgium) and signal intensity was measured using Image ${ }^{\circledR}$ software (by Wayne Rasband at $\mathrm{NIH}$, Bethesda, Maryland, USA).

\section{Caspase activity assay}

Cells were seeded into white-walled 96-well plates with a clear and flat bottom. After $24 \mathrm{~h}$ of incubation, cells were treated with the indicated concentrations of WEHI539 and incubated for another $48 \mathrm{~h}$. For measuring the activities of Caspases 3/7, 8, and 9 the luminescencebased Caspase-Glo Assay Kits from Promega (Madison, WI, USA) have been used, according to the manufacturer 's protocol. Luminescence of each sample was quantified in a plate-reading luminometer (Infinite 200 PRO; Tecan, Männedorf, Switzerland).

\section{Quantification of cell death by flow cytometry}

Cells were seeded and treated as indicated for $48 \mathrm{~h}$. Subsequently, supernatants were transferred to FACS tubes and cells were gently detached using Accutase (PAA Laboratories) and transferred into the tubes as well. After centrifugation $(200 \times g, 5 \mathrm{~min})$, cells were resuspended in a hypotonic buffer containing $0.1 \%(\mathrm{w} / \mathrm{v})$ sodium citrate, $0.1 \%(\mathrm{v} / \mathrm{v})$ Triton $\mathrm{X}-100$ and $50 \mu \mathrm{g} / \mathrm{ml}$ propidium iodide (all from Sigma-Aldrich). After $1 \mathrm{~h}$ of incubation in the dark at $4{ }^{\circ} \mathrm{C}$, total DNA content of cells was measured according to the protocol of Nicoletti et al. by FACS analysis ${ }^{34}$, using the FACS Diva 6 and the FlowJo 7.6.5. software (BD Biosciences, San José, CA, USA). Cells in the sub-G1 fraction were considered apoptotic.

\section{siRNA transfection}

Cells were seeded, cultured for $24 \mathrm{~h}$ and transfected by using Lipofectamine RNAiMAX (Thermo Fisher Scientific, Waltham, MA, USA) in OptiMEM cell culture medium without any supplements (Gibco, Karlsruhe, Germany), according to the manufacturer's protocol. The following siRNA sequence, targeting the BCL-XL (BCL2L1) mRNA was applied (Eurofins Scientific, Luxembourg): $5^{\prime} \rightarrow 3^{c}$ GCUUGGGAUAAAGAUGCAA (sense) and $5^{\prime} \rightarrow 3^{\prime}$ UUGCAUCUUUAUCCCAAGC (antisense). As a control served the following scrambled siRNA: $5^{c} \rightarrow 3^{c}$ AGACCCACUCGGAUGUGAAGAGAUA (sense) and $5^{\prime} \rightarrow 3^{\prime} \quad$ UAUCUCUUCACAUCCGAGUGGGUCU (antisense).

\section{Mice and AOM/DSS model}

C57BL/6 mice were purchased from Charles River Laboratories (Wilmington, MA, USA) and housed in individually ventilated cages at the SPF animal facility of the Interfaculty Biomedical Research Facility (IBF), University of Heidelberg, Germany. Animals were kept under a $12 \mathrm{~h}$ light cycle with ad libitum feeding and all experiments on mice were conducted according to institutional, national and European animal regulations with all protocols approved by local government authorities and a daily review of the health status of the animals was done. The health status was gathered using a scoring system, which comprised diarrhea severity, weight loss, rectal prolapse, apathy and aggressiveness as a sign for pain.

28 gender-matched mice (to account for possible dropouts during tumor induction) with an age of ten weeks and a body weight $>20 \mathrm{~g}$ were injected intraperitoneally with $10 \mathrm{mg}$ per $\mathrm{kg}$ body weight of the carcinogenic agent azoxymethane (AOM; Sigma-Aldrich). Subsequently, intestinal tumor formation was promoted by three cycles of the proinflammatory reagent dextran sodium sulfate (DSS; MP Biomedicals, Santa Ana, CA, USA) in the drinking water $(2 \% \mathrm{w} / \mathrm{v})$. Each cycle lasted 7 days with 14 days of recovery in between. After one week of recovery, subsequent to the last DSS cycle, animals were randomly divided into four groups ( $n=5$ per group) and treated as follows:

Group I: daily by oral gavage with $25 \mathrm{mg} / \mathrm{kg}$ of the BCLXL inhibitor A-1331852, dissolved in 60\% Phosal 50 PG (Lipoid, Ludwigshafen, Germany), 27.5\% polyethylene glycol 400 (Sigma-Aldrich), 10\% ethanol, and 2.5\% dimethyl sulfoxide (DMSO; Serva, Heidelberg, Germany) and with a formulation as described ${ }^{35}$. Group II: three times a week with $30 \mathrm{mg} / \mathrm{kg} 5 \mathrm{FU}$, dissolved in saline, by intraperitoneal injection. Group III: with the combination of A-1331852 and 5FU. Group IV: with the respective solvents as a control. For evaluation of diarrhea severity and anal bleeding during tumor induction and treatment, a score was used as previously described ${ }^{25}$. After 14 days of treatment, blood was collected from the retrobulbar venous sinus and mice were sacrificed by cervical dislocation with subsequent opening of their bowel cavity. Colons were removed, rinsed with PBS and opened longitudinally. Tumors were counted and tumor diameters were measured with a sliding caliper by two blinded investigators (ALS and NS). Tumor volumes were calculated from the measured diameters with the following equation: $\frac{4}{3} * \pi * r^{3}$. The blood was transferred to the Diagnostic Center of the Heidelberg University Hospital for compilation of a small hemogram, according to standard procedures.

\section{Immunohistochemistry}

Mouse colon tissues were isolated, rinsed with PBS, covered with OCT mounting medium (Science Services, Munich, Germany) and gradually frozen in the gas phase of liquid nitrogen. Eight-micrometer cryosections were cut (Cryostat, Thermo Fisher Scientific) and fixed in $4 \%$ paraformaldehyde (PFA), followed by heat induced antigen retrieval with citrate buffer (pH6). Subsequently, staining was performed by using the NovoLink Polymer Detection System (Leica Microsystems, Wetzlar, 
Germany), according to the manufacturer's protocol. The following primary antibodies were used for murine tissues: BCL-XL (\# 2764, Cell Signaling Technology), Ki67 and cleaved PARP (\# ab16667 and \# ab32064, Abcam, Cambridge, UK).

Paraffin-embedded human tissue sections were dewaxed and rehydrated using xylene and a series of graded alcohols, followed by antigen retrieval and staining with antibodies against BCL-XL (\# 2764, Cell Signaling Technology) or cleaved PARP (\#5625, Cell Signaling Technology), as described above.

Staining intensity was evaluated blinded for the groups by two independent and experienced examiners by utilizing a scoring system in which values for staining quantity $(1-10 \%=1,11-50 \%=2,51-80 \%=3,81-100 \%$ $=4$ ) and quality (unstained $=0$, weak $=1$, moderate $=2$, strong $=3$ ) were allocated and multiplied in the end ${ }^{36}$. Negative controls were generated by omitting the primary antibody.

\section{xCELLigence real-time cell analysis}

The xCELLigence real-time cell analysis (RTCA) instrument (ACEA Biosciences, San Diego, CA, USA) allows continuous monitoring of cell proliferation and viability by measuring electrical impedance. Therefor, 7500 CCD $841 \mathrm{CoN}$ cells in $100 \mu \mathrm{l}$ medium per well were seeded in gold-plated 96-well E-Plates (ACEA Biosciences). Subsequently, cells were monitored with a time interval of $5 \mathrm{~min}$ for a total of $72 \mathrm{~h}$. $24 \mathrm{~h}$ after seeding, cells were treated as indicated and curves were normalized at this point. Impedance was calculated by the RTCA software and reported as Cell Index (CI), as described previously ${ }^{37}$.

\section{Patients and human tissue culture}

Primary CRC tissues $(n=27)$ were obtained by surgical resection in the Department of General and Transplantation Surgery, University of Heidelberg, Germany. The usage of patient tissue for research purposes was approved by the local ethics committee of the University Hospital of Heidelberg. All analyses were done anonymously and written informed consent was obtained from all donors (S-443/2013 and S-649/2012). This study was conducted in accordance with the Declaration of Helsinki. The cohort included patients diagnosed in all UICC stage (I-IV), $70 \%$ of the tumors were colon carcinomas and $30 \%$ rectal carcinoma.

Human primary CRC tissues were processed according to the protocol of Georg Gdynia and colleagues ${ }^{36}$. In brief, tumor tissues were cut in Krebs-Henseleit buffer into $300 \mu \mathrm{m}$ thick slices using a vibratome (Leica Biosystems, Wetzlar, Germany), according to manufacturer's protocol. Afterwards, tissue specimens were transferred onto filter membrane inserts and placed in six-well plates (both
Corning, Corning, NY; USA). From below, wells were gently filled with about $1.5 \mathrm{ml}$ DMEM culture medium (Gibco), supplemented with 10\% heat-inactivated fetal calf serum (PAA Laboratories), 1\% penicillin/streptomycin (PAA Laboratories) and the respective inhibitor or chemotherapeutic agent as indicated, until the lower surface of the membrane was reached. Tissue specimens were kept at the air-liquid interface for $48 \mathrm{~h}$ in a humidified atmosphere at $37^{\circ} \mathrm{C}$ and $5 \% \mathrm{CO}_{2}$. Finally, tissue slices were fixed in $10 \%$ formalin and paraffin embedded. Twomicrometer sections were stained with Hematoxylin and Eosin (H\&E) for blinded assessment of tissue viability by a trained pathologist (GG).

\section{Statistical analysis}

Statistical analysis was performed using R (version 3.5.1; r-project.org) and GraphPad Prism 8.2.1 (GraphPad; San Diego, CA, USA). All in vitro experiments were performed in at least triplicates and data are presented as mean $+\mathrm{SD}$. Obtained data were submitted to analysis of variance (ANOVA) with the post-hoc Tukey multiple comparison test or by two-way repeated measures analysis of variance (two-way ANOVA) followed by Dunnett's multiple comparison test. $P$ values $<0.05$ were considered significant and were indicated as following: ${ }^{*} P<0.05$, ** $P$ $<0.01,{ }^{* * * *} P<0.001$.

\section{Results}

\section{$\mathrm{BCL}-\mathrm{XL}$ is highly active in colorectal cancer}

Pan-cancer RNA sequencing cohorts enable the comparative transcriptomic analysis of biological networks, pathways, and genes of interest. Since mRNA levels not necessarily correlate with protein activity, we used the metaVIPER algorithm to infer the activity of antiapoptotic BCL-2 proteins from RNA sequencing data. This algorithm was designed to assess the activity of a protein by an integrative analysis of its transcriptional targets, i.e., its interactome ${ }^{29}$. In this way, RNA sequencing data cannot only reveal changes on the transcriptional level but also posttranslational effects.

Comparing the estimated protein activity of BCL-2, BCL-XL, and MCL-1 within a CRC cohort $(n=68)$ revealed only BCL-XL but not BCL-2 or MCL-1 to be highly active (Fig. 1a). Moreover, protein activity of BCL2 was inversely correlated with that of BCL-XL $(r=$ $-0.68)$, while there was no correlation between MCL-1 and BCL-XL (Fig. 1b). Estimating the protein activity of BCL-2, BCL-XL, and MCL-1 within the entire NCT/ DKTK MASTER cohort $(n=1521)$ showed distinct differences between entities, with CRC showing one of the highest activities of BCL-XL (Fig. 1c). In contrast, estimated protein activities of $\mathrm{BCL}-2$ and $\mathrm{MCL}-1$ were relatively low in CRC compared to the complete cohort (Fig. 1d, e). 


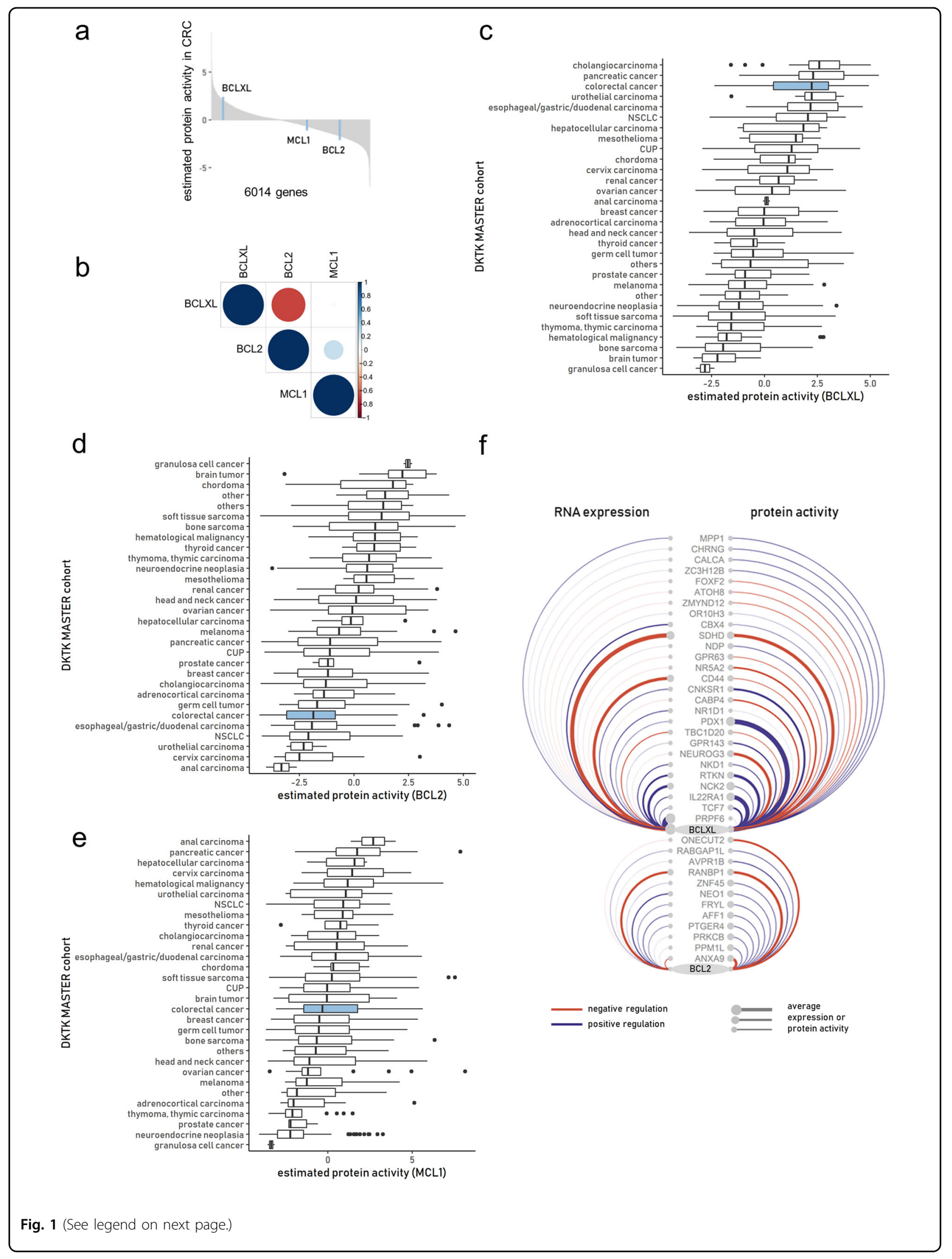


(see figure on previous page)

Fig. 1 Pan-cancer protein activity of BCL-XL, BCL-2, and MCL-1. a Waterfall plot of the ranked 6014 master regulators identified in the CRC cohort $(n=68)$ by metaVIPER algorithm. b Heatmap of correlation coefficients between estimated protein activities of BCL-XL, BCL-2, and MCL-1 across the CRC cohort $(n=68)$. c-e Ranking of tumor entities across the NCT/DKTK MASTER cohort $(n=1521)$ according to the estimated median protein activity of BCL-XL (c), BCL-2 (d), and MCL-1 (e) obtained by metaVIPER algorithm. $\mathbf{f}$ Arc diagram of the regulatory network of BCL-XL and BCL2. The edges and nodes were color-coded according the direction of regulation and weighted according the average expression or protein activity in the CRC subset of the NCT/DKTK MASTER cohort.

Comparing RNA expression (transcripts per million, tpm), and not estimated protein activity, across the cohort, BCL-XL again ranked highest among the antiapoptotic BCL-2 proteins investigated in CRC. Intriguingly, this rank did not match the estimated protein activity, since it showed a comparably lower transcript level, indicating a posttranslational effect (Fig. S1).

The cause for the discrepancy between the expression and estimated protein activity of the BCL- 2 proteins can be visualized by the weight of positive and negative regulators in the CRC subset of the MASTER cohort (Fig. 1f). By incorporating the information about the interactome, more regulators are identified that ultimately influence the protein activity of BCL-XL and BCL-2.

\section{Inhibition of $\mathrm{BCL}-\mathrm{XL}$ induces apoptosis in human colorectal cancer cells}

In order to evaluate the efficiency of target-specific BH3-mimetics in inducing CRC cell death, four human CRC cell lines were treated with WEHI-539 (BCL-XL inhibitor), ABT-199 (BCL-2 inhibitor), and S63845 (MCL-1 inhibitor) in three different concentrations (Fig. 2a). Strikingly, all tested cell lines were most sensitive towards inhibition of BCL-XL ([Colo205: WEHI-539: $1 \mu \mathrm{M} p=\mathrm{ns}, 5 \mu \mathrm{M}$ and $20 \mu \mathrm{M} p<0.0001$; ABT-199: all concentrations $p=\mathrm{ns}$; S63845: $1 \mu \mathrm{M} p=\mathrm{ns}, 5 \mu \mathrm{M} p=$ 0.0002, $20 \mu \mathrm{M} p<0.0001$ ]; [HT29: WEHI-539: $1 \mu \mathrm{M} p=$ ns, $5 \mu \mathrm{M} p=0.0004,20 \mu \mathrm{M} p<0.0001$; ABT-199 and S63845: all concentrations $p=\mathrm{ns}]$; [CaCo2: WEHI-539: $1 \mu \mathrm{M} p=0.0298,5 \mu \mathrm{M}$ and $20 \mu \mathrm{M} p<0.0001$; ABT-199 and S63845: all concentrations $p=\mathrm{ns}$ ] and [SW480: WEHI-539: $1 \mu \mathrm{M}, 5 \mu \mathrm{M}$ and $20 \mu \mathrm{M} p<0.0001$; ABT-199: $1 \mu \mathrm{M}$ and $5 \mu \mathrm{M} p=\mathrm{ns}, 20 \mu \mathrm{M} p<0.0001$; S63845: all concentrations $p=\mathrm{ns}]$ ). However, the degree of cell death induction varied greatly among the different cell lines. Subsequent western blot analyses with antibodies detecting $\mathrm{BCL}-\mathrm{XL}, \mathrm{MCL}-1$, and $\mathrm{BCL}-2$ revealed that susceptibility to BCL-XL inhibition strongly depends on its basal expression level, showing a linear correlation with $\mathrm{R}^{2}=0.91$ (Fig. 2b, c). Neither for MCL-1 nor for BCL-2 such a correlation was observed (Fig. S2).

To evaluate the mode of cell death induced by BCL-XL inhibition, luminescence assays measuring the activation of Caspases 3/7, 8, and 9 were done (Fig. 2d). These experiments were performed in SW480, representing a cell line with high BCL-XL expression and inhibitor susceptibility, as well as in HT29 cells, representing a cell line with low expression of BCL-XL. In both cell lines, the luminescence substrate assays revealed a dose-dependent increase of Caspase 9 and Caspase 3 activation and a minor but in HT29 cells still significant activation of Caspase 8 . Since Caspase 8 is not only activated in the extrinsic pathway but can also be cleaved by Caspase 3 in a feed-forward loop, enhancing apoptosis signaling ${ }^{38,39}$, the observed combination indicates apoptosis induction via the intrinsic pathway.

\section{Inhibition of BCL-XL significantly enhances the effectiveness of chemotherapeutic agents in vitro}

To test the response under treatment with chemotherapeutic agents 5-fluorouracil (5FU) and irinotecan, which are commonly used in treatment regimens for CRC patients, the four CRC cell lines were incubated with equal doses of the respective cytostatic drug and cell death was subsequently quantified by FACS analysis. In this setting, the resulting cell death increase was inversely correlated with basal expression of BCL-XL, with similar results for $5 \mathrm{FU}\left(\mathrm{R}^{2}=0.79, \log\right.$-transformed predictor $)$ and irinotecan $\left(R^{2}=0.93\right.$, log-transformed predictor), even though the two chemotherapeutics employ different modes of action in the context of cell death induction (Fig. 3a). Since a relatively high basal BCL-XL expression level seemed to render cells susceptible towards inhibitor treatment and resistant towards chemotherapy, we combined these two treatment strategies in HT29 and SW480 cells. For both cell lines, inhibitor doses as well as cytostatic drug concentrations were individually adjusted to induce a moderate cell death increase of $5-15 \%$ under mono-drug treatment. Strikingly, the additional chemical inhibition of BCL-XL significantly enhanced the effectiveness of irinotecan (Fig. 3c; upper panels; $p<0.0001$ for HT29 and SW480) and 5FU (lower panels; $p<0.0001$ for HT29 and $p=0.0015$ for SW480) in both cell lines. Western blot analyses of BCL-XL, MCL-1, and BCL-2 expression levels under WEHI-539 treatment showed decreased expression of BCL-XL in HT29 cells in presence of the inhibitor (Fig. 3b). Nevertheless, HT29 cells strongly responded to combined treatment with BCL-XL inhibitor and chemotherapy. In SW480 cells, no differences in the expression levels of the mentioned 


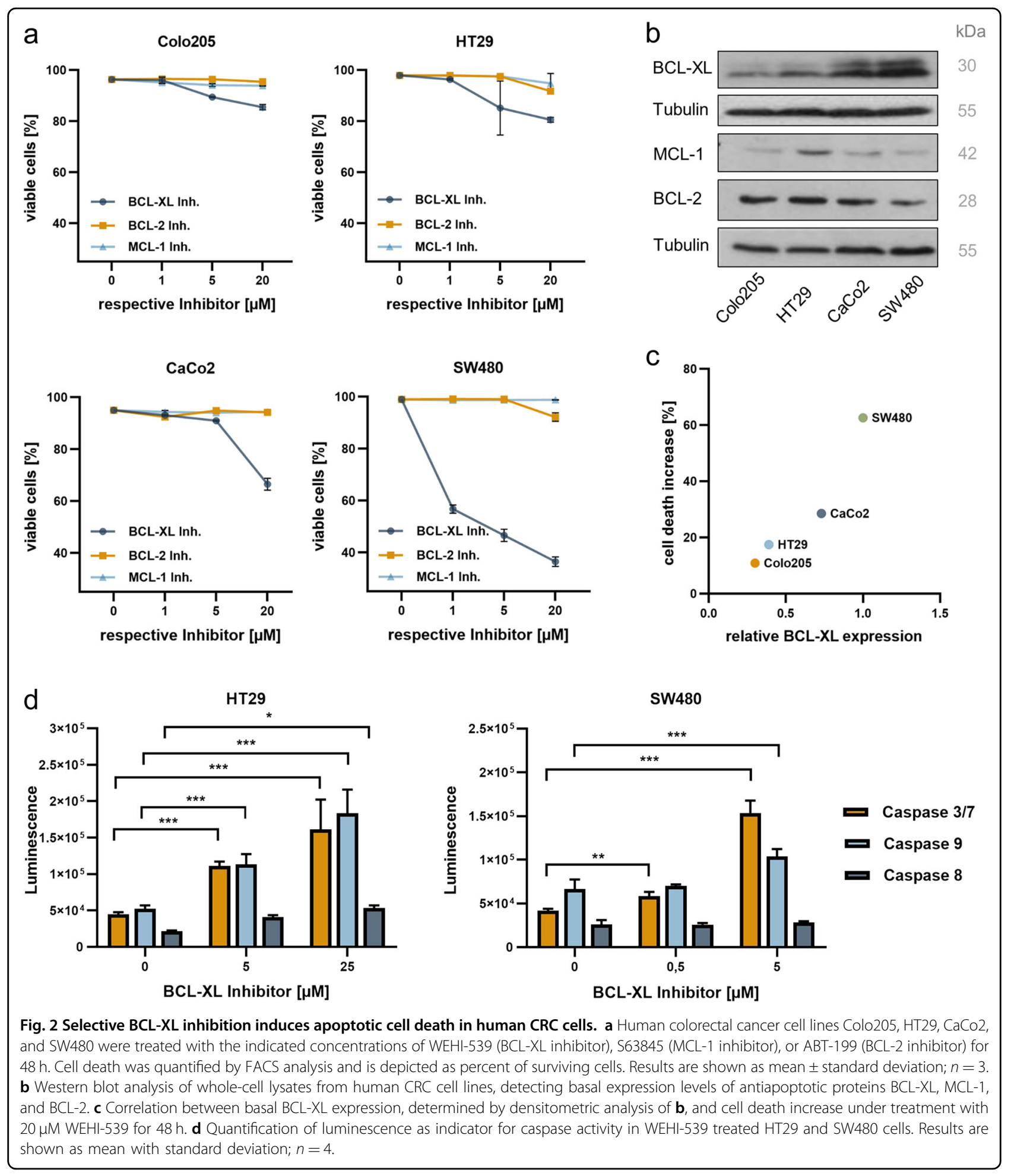

antiapoptotic proteins were observed under inhibitor treatment. In order to prove target specificity, the combination experiment was repeated applying siRNAmediated BCL-XL (BCL2L1) silencing instead of chemical inhibition. Effectiveness of transfection was validated by western blot analysis (Fig. S2b). Again, a significantly increased response to chemotherapeutic agents irinotecan (Fig. 3d; upper panels) and 5FU (lower panels) was observed in both cell lines upon knockdown of BCL-XL. 


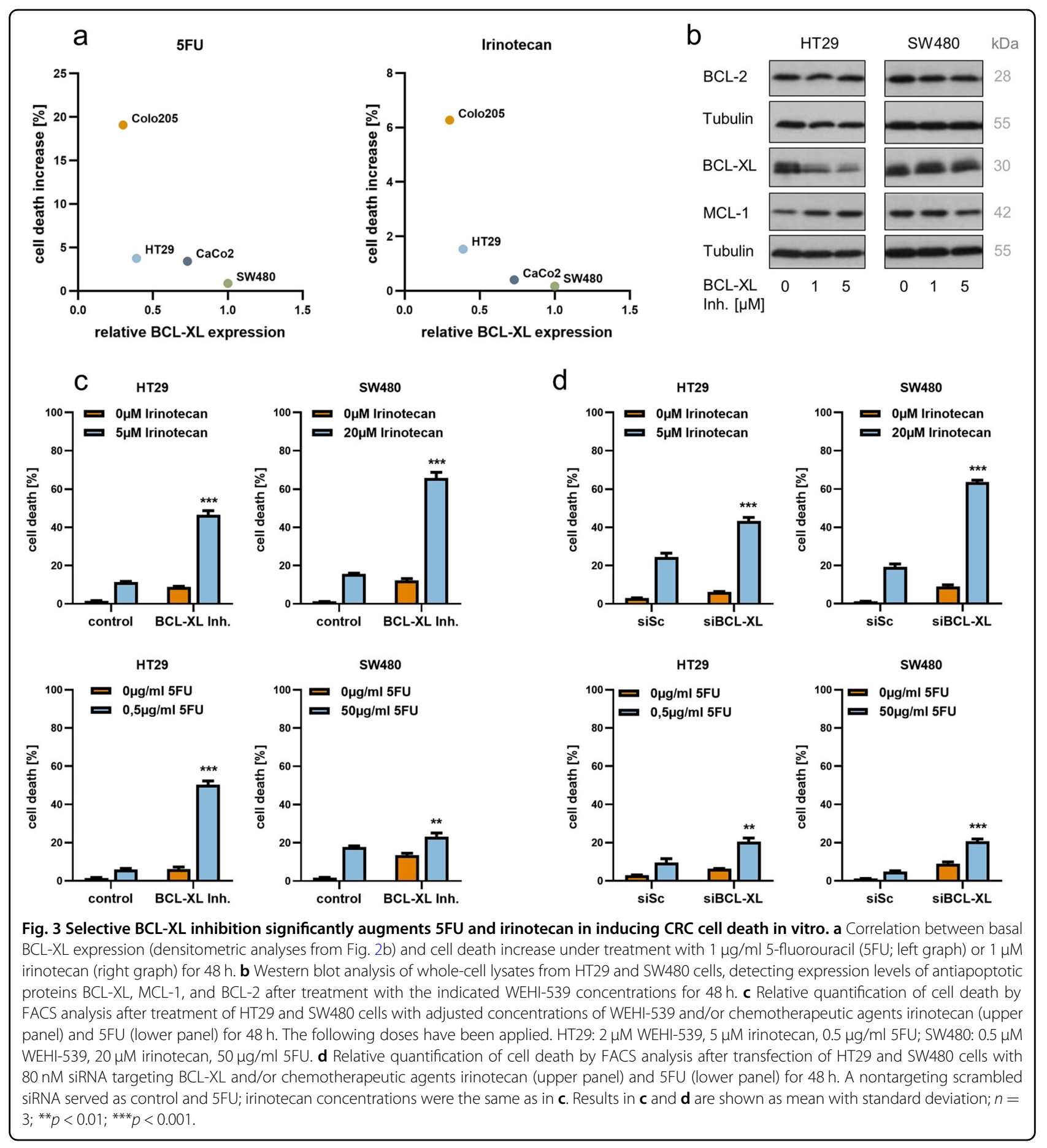

\section{Chemical inhibition of BCL-XL is effective in the treatment of colorectal tumors in vivo}

In order to examine whether colorectal tumors respond to a systemically applied BCL-XL inhibitor, a chemically induced CRC mouse model was utilized. Specifically, we aimed to compare responses to BCL-XL inhibitor treatment with responses to $5 \mathrm{FU}$, which is an essential part of standard of care treatment regimens for CRC, and whether addition of a BCL-XL inhibitor can enhance the effectiveness of $5 \mathrm{FU}$, as observed in vitro. Furthermore, we sought to explore the safety and toxicity profile of such a combination therapy in vivo. For the mouse experiments A-1331852, which is a WEHI-539 related but orally available BCL-XL inhibitor, was used.

For induction of colorectal tumors, the combination of $\mathrm{AOM}$ and DSS was administered ${ }^{40}$. Subsequently, mice 


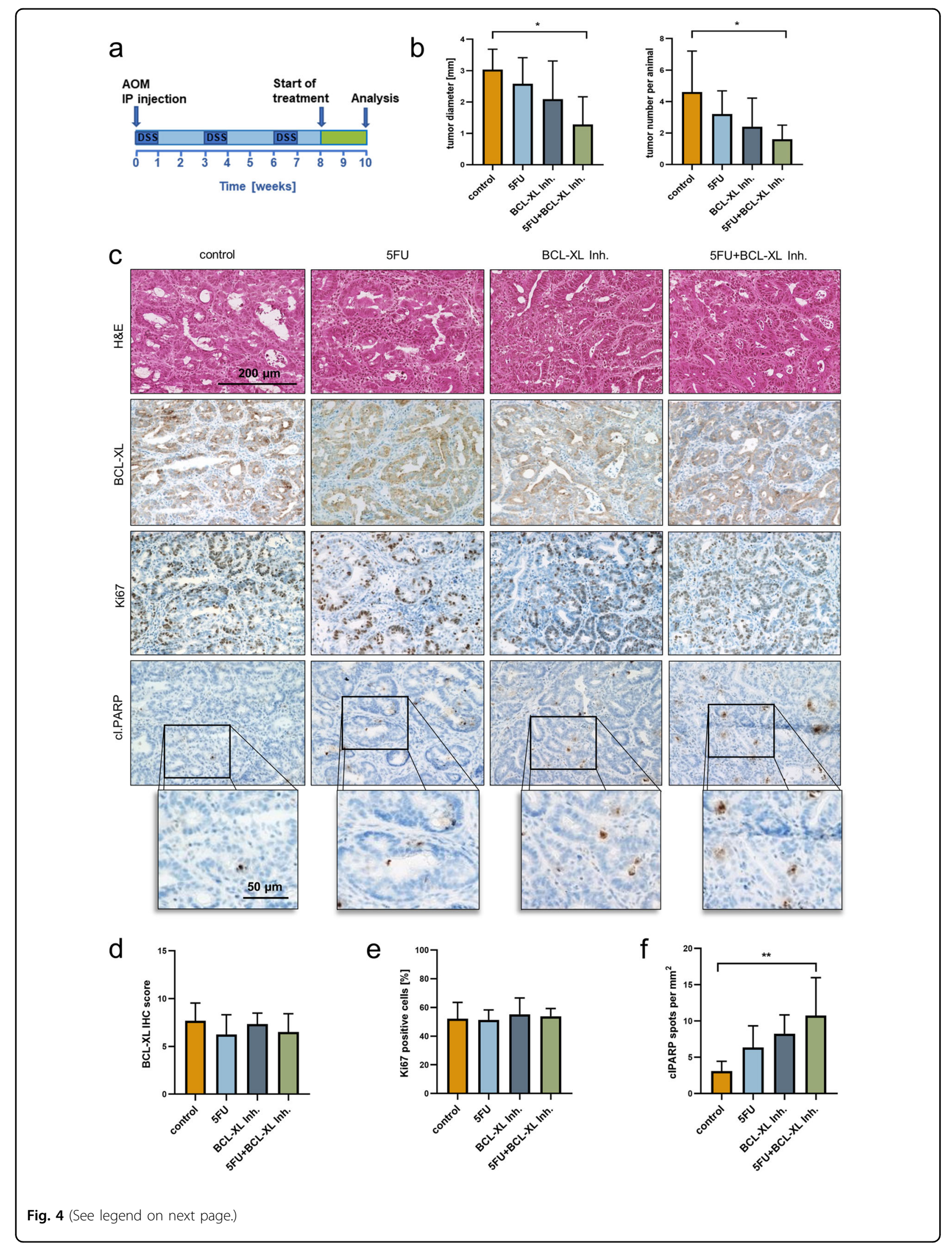


(see figure on previous page)

Fig. 4 Selective BCL-XL inhibition is effective in the treatment of tumors in a murine CRC model. a Schematic time course with a tumor initiation and a treatment phase. Initiation: intraperitoneal injection of azoxymethane (AOM) at the start day and three cycles of dextran sodium sulfate (DSS) in the drinking water ( $2 \% \mathrm{w} / \mathrm{v}$ ). Treatment: $25 \mathrm{mg} / \mathrm{kg} \mathrm{A}-1331852$ (orally available BCL-XL inhibitor) daily by oral gavage or $3 \times$ per week $30 \mathrm{mg} / \mathrm{kg}$ 5FU by intraperitoneal injection for a total time period of 14 days. b Tumor sizes (left graph) and numbers (right graph) in mice after treatment with A-1331852, 5FU, the combination of both or the respective solvents as a control ( $n=5$ animals per group). c Hematoxylin and Eosin (H\&E) staining (upper panel) and immunohistochemical staining against BCL-XL, Ki67 (proliferation marker) and cleaved PARP (cI.PARP; apoptosis marker) on tumors derived from animals treated as depicted in $\mathbf{a}$. Scale bars as indicated $\mathbf{d}$ Scoring of the $I H C$ staining of BCL-XL depicted in $\mathbf{c}$. e Percentage of Ki67-positive cells, referred to Hematoxylin stained nuclei. $\mathbf{f}$ Number of cl.PARP positive spots per $\mathrm{mm}^{2}$. Results in $\mathbf{b}, \mathbf{d}, \mathbf{e}$, and $\mathbf{f}$ are shown as mean with standard deviation; ${ }^{*} p<0.05,{ }^{* *} p<0.01$.

received either the BCL-XL inhibitor $(25 \mathrm{mg} / \mathrm{kg}$, daily by oral gavage), $5 \mathrm{FU}(30 \mathrm{mg} / \mathrm{kg}$, $3 \times$ per week by i.p. injection), the combination of both or solely the respective solvents as a control for 2 weeks, and afterwards tumor numbers and diameters were determined. Measurement of tumor diameters revealed that both, treatment with 5FU (mean tumor diameter: $2.58 \mathrm{~mm}$ ) as well as treatment with the inhibitor $(\varnothing 2.09 \mathrm{~mm})$, led to apparent, albeit not significant tumor shrinkage compared to controls $(\varnothing 3.04 \mathrm{~mm}$ ). However, the strongest effect was observed upon combined treatment. With a mean tumor diameter of $1.3 \mathrm{~mm}$ (Fig. 4b; left graph; $p=0.021$ ) we observed in this group a significant decrease of tumor volume to $14 \%$, if compared to controls (calculated tumor volumes are depicted in Fig. S3a). Furthermore, animals that received either $5 \mathrm{FU}$ or $\mathrm{BCL}-\mathrm{XL}$ inhibitor treatment displayed lower tumor numbers than the controls. Adding the BCL-XL inhibitor to 5FU caused the strongest effect, proving the value of BCL-XL inhibition in combination with the fluoropyrimidine (Fig. 4b; right graph; $p=0.047$ ).

To investigate whether BCL-XL expression is decreased in tumors derived from mice that received the inhibitor alone or the combination therapy, immunohistochemical staining for BCL-XL was performed (Fig. 4c). The staining revealed an unaltered expression of $\mathrm{BCL}-\mathrm{XL}$ in these two groups compared to the control and the $5 \mathrm{FU}$ treated groups (Fig. 4d). In a second step, we sought to analyze if the observed differences in tumor size and numbers in the different groups rely on an altered proliferation activity or on cell death induction in the tumors. While we found the level of Ki67-positive cells unalteredly high (Fig. 4c, e), staining for cleaved PARP (cl.PARP), a Caspase substrate, revealed considerable apoptosis induction in tumors derived from treated animals but not in the controls. The highest cl.PARP positivity was present in tumors derived from the group that underwent combinatorial treatment with $5 \mathrm{FU}$ and the BCL-XL inhibitor (Fig. $4 \mathrm{c}, \mathrm{f} ; p=0.006$ ).

\section{Chemical inhibition of BCL-XL does not affect nontransformed intestinal epithelial cells}

By immunohistochemical staining of tumor-free mucosa with antibodies detecting Ki67 and cl.PARP, we found the proliferative capacity of nontransformed intestinal epithelial cells to be marginally decreased and the viability to be unaltered in mice after treatment with $5 \mathrm{FU}$, the BCL-XL inhibitor or the combination of both (Fig. 5a and for quantification Fig. S3b). In addition, the chosen doses of $5 \mathrm{FU}$ and the inhibitor or their combined application did not induce significant weight loss during the 2 weeks of treatment (Fig. 5b). To further test whether intestinal epithelial cells are less prone to cell death induction via chemical BCL-XL inhibition compared to CRC cells, nontransformed human intestinal epithelial cells (CCD $841 \mathrm{CoN}$ cell line) were treated with $20 \mu \mathrm{M}$ WEHI-539, $20 \mu \mathrm{M}$ ABT-199, or $20 \mu \mathrm{M}$ S63845, representing the highest doses used for screening in human CRC cell lines (Fig. 2a). Two-micromolar Staurosporine were used to generate a positive control for cell death induction. For the measurement, the xCELLigence system that allows real-time monitoring of proliferation and viability has been used. The benefit of this system is a non-invasive, label-free and close monitoring of growth inhibition or cell death induction of adhesive cells under treatment. The device uses gold-plated microelectrodes to measure electrical impedance from which the Cell Index (CI) as unitless parameter is calculated ${ }^{37}$. Hereby, we found that none of the inhibitors significantly induced cell death in CCD 841 CoN cells (Fig. 5c).

To further assess putative adverse effects on blood cells, blood was collected after treatment with 5FU, the BCL-XL inhibitor, or the combination of both. We found no significant changes in leukocyte numbers; however, thrombocyte and erythrocyte counts were significantly decreased in mice treated with the BCL-XL inhibitor alone or in combination with 5FU (Fig. 5d; [the inhibitor lowered thrombocytes to $60 \% ; p=0.0026$ and the combination to $48 \% ; p=0.0005$ ]; [the inhibitor lowered erythrocytes to $80 \% ; p=0.034$ and the combination to $65 \%$; $p=0.001]$ all comparisons were made to the respective controls). Despite a known effect on hematopoiesis, 5FU alone did neither lead to a significant decrease of thrombocytes nor erythrocytes. Additional application of 5FU to BCL-XL inhibition aggravated the phenotype observed under inhibitor-only treatment. In order to analyze the reason for the decreased number of erythrocytes in further detail, we additionally determined 

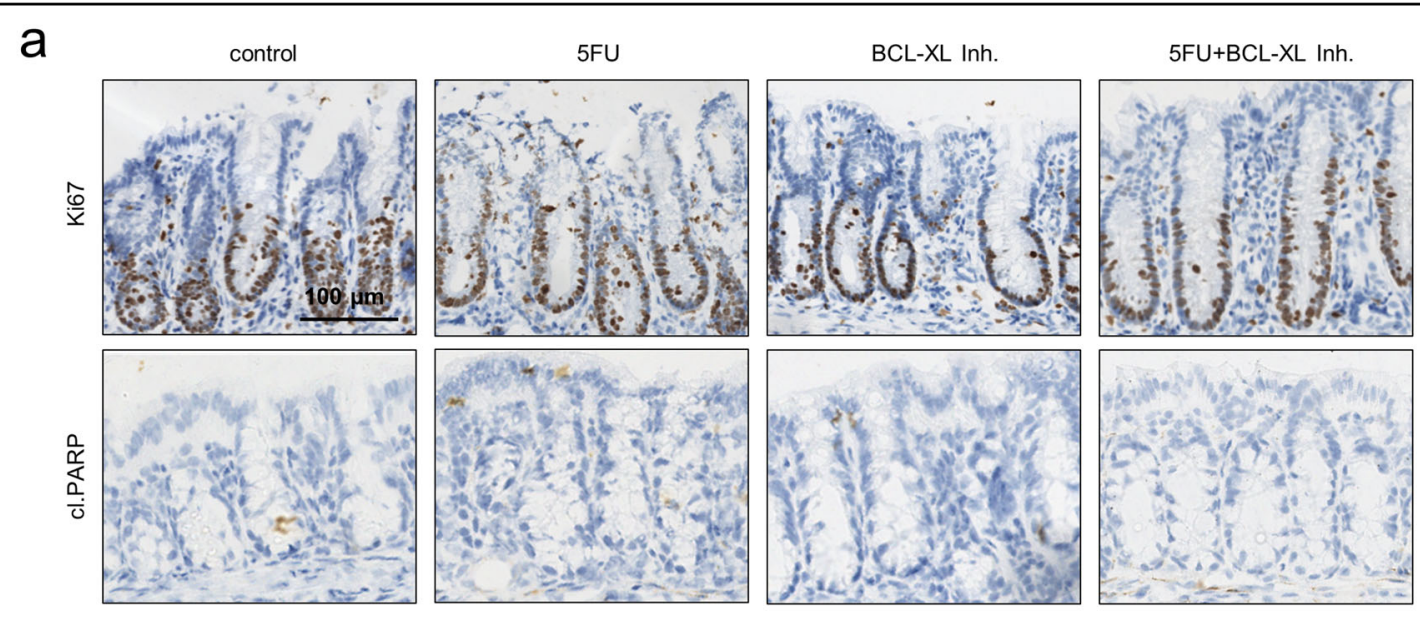

b
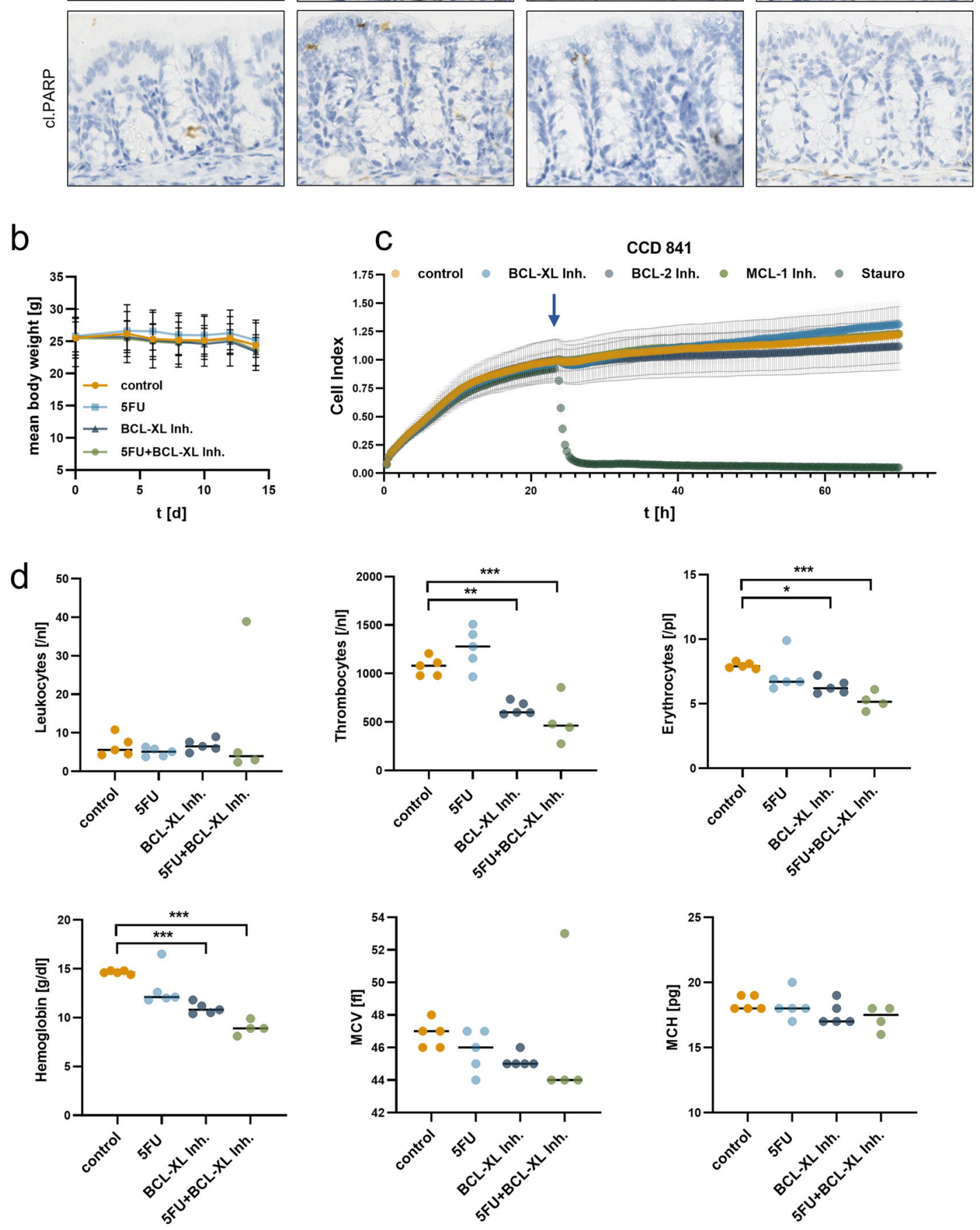

Fig. 5 (See legend on next page.) 
(see figure on previous page)

Fig. 5 Selective BCL-XL inhibition does not induce cell death in intestinal epithelial cells. a Immunohistochemical staining against Ki67 (proliferation marker) and cleaved PARP (cl.PARP; apoptosis marker) on longitudinal sections of colon crypts, derived from animals treated as depicted in (4a). Scale bar indicates $100 \mu \mathrm{m}$. b Body weight alterations during the course of treatment. c Impedance monitoring of human intestinal epithelial cell line CCD 841 CoN for 72 h. After $24 \mathrm{~h}$ (blue arrow), cells were treated with 20 4M of WEHI-539 (BCL-XL inhibitor), S63845 (MCL-1 inh.) or ABT-199 (BCL-2 inh.). DMSO was used as vehicle control and $2 \mu \mathrm{M}$ Staurosporine (Stauro) as positive control for cell death induction. Results are shown as mean \pm standard deviation; $n=3$. $\mathbf{d}$ Small hemogram from mice after indicated treatment. MCV mean corpuscular volume, MCH mean corpuscular hemoglobin. Results are shown as median; ${ }^{*} p<0.05$; ${ }^{* *} p<0.01$; ${ }^{* * *} p<0.001$.

hemoglobin levels, the average erythrocyte size (mean corpuscular volume, MCV) and the amount of hemoglobin per red blood cell (mean corpuscular hemoglobin, $\mathrm{MCH})$. The analyses revealed significantly decreased overall hemoglobin levels after inhibitor treatment (inhibitor: $p=0.0003$; combination: $p<0.0001$ ) but an unaltered hemoglobin content per erythrocyte. In combination with the slightly decreased MCV values in the inhibitor-treated groups, this demonstrated the occurrence of microcytic anemia.

\section{Chemical inhibition of BCL-XL in combination with 5FU significantly decreases tumor cell viability in human colorectal cancer specimens ex vivo}

In order to transfer our in vitro and in vivo findings into a more translational setting, we utilized tissue cultures of patient-derived CRC specimens. To this end, CRC samples from patients who underwent surgery $(n=27)$ were freshly cut and treated for $48 \mathrm{~h}$. Patient characteristics regarding age, gender, UICC stage and tumor localization are itemized in supplementary table 1 . From each patient sample, several slices were taken and treated with either $50 \mu \mathrm{g} / \mathrm{ml} 5 \mathrm{FU}, 5 \mu \mathrm{M}$ WEHI-539, the combination of both or solely with the respective solvents as a control, by supplementing the medium (Fig. 6a). H\&E staining with subsequent histomorphologic analysis revealed an only minor decrease of tumor cell viability under single agent therapy but a significant loss of viability under the combinatorial treatment (Fig. 6b, c; $p=0.023$ ). This finding was confirmed by immunohistochemical staining of cleaved PARP, which showed that the combination of $5 \mathrm{FU}$ and the BCL-XL inhibitor led to a significant increase of the apoptotic cell death level (Fig. 6b, d; $p=0.039$ ). In-line with our in vivo findings, immunohistochemical staining of BCL-XL revealed no significant expression differences under treatment (Fig. 6b, e).

\section{Discussion}

Since avoidance of apoptosis is a general hallmark of tumors, cell death preventing factors, such as antiapoptotic members of the BCL-2 protein family and their potential inhibition, are in the focus of cancer research ${ }^{41}$. Thus, there is a pending medical need for evaluating the effectiveness and safety of novel and selective BH3- mimetics, especially in solid tumors, which urged us to further delineate their potential in the context of CRC treatment.

Emerging concepts based on functional studies suggest a tissue and organ specific rather than redundant function of antiapoptotic Bcl-2 proteins ${ }^{42-44}$. Few studies have investigated the specific interactome of Bcl-2 proteins in certain tumor subtypes ${ }^{43,44}$.

In previous work we had identified $\mathrm{BCL}-\mathrm{XL}$ as the only antiapoptotic BCL-2 protein that is overexpressed in $\mathrm{CRC}^{25}$. In-line, $\mathrm{CRC}$ shows one of the highest frequencies of BCL2L1 (gene coding for BCL-XL) amplifications across The Cancer Genome Atlas (TCGA) database ${ }^{45}$. By using a network biology-based approach, we were now for the first time able to derive protein activity of BCL-2, BCL-XL, and MCL-1 in a pan-cancer cohort (NCT/ DKTK MASTER cohort). Protein activity correlates with mRNA expression in most cases. Estimated protein activity by the MVIPER algorithm summarizes the expression of transcriptional targets of each protein in the regulon leading to an estimated high protein activity in a couple of cases with low mRNA expression and vice versa. We could show that CRC ranks on the third place among all investigated tumor entities regarding estimated BCL$\mathrm{XL}$ activity. Interestingly, a ranking of tumor entities across the pan-cancer cohort according to transcript levels of BCL-XL led to a slightly different positioning of CRC. This could point to a stabilization of the protein in CRC. Since a decreased expression of proapoptotic members of the BCL-2 protein family would have a similar functional outcome, this effect could also be caused by a shift in the balance between anti- and proapoptotic proteins towards enhanced BCL-XL activity ${ }^{46}$. This finding underlines the usefulness of estimating protein activity in addition to RNA-seq.

In-line with the above mentioned findings, human CRC cell lines were found to be highly sensitive towards selective inhibition of BCL-XL but not MCL-1 or BCL-2, even though binding affinity of WEHI-539 is with a dissociation constant $\left(\mathrm{K}_{\mathrm{D}}\right)$ of $0.6 \mathrm{nM}$ lower than that of $\mathrm{S} 63845\left(K_{\mathrm{D}}=0.19 \mathrm{nM}\right)^{47,48}$. Noticeably, we found that the susceptibility towards WEHI-539 treatment correlated with the basal expression level of BCL-XL. This coincides with a previous study utilizing various human CRC cell 

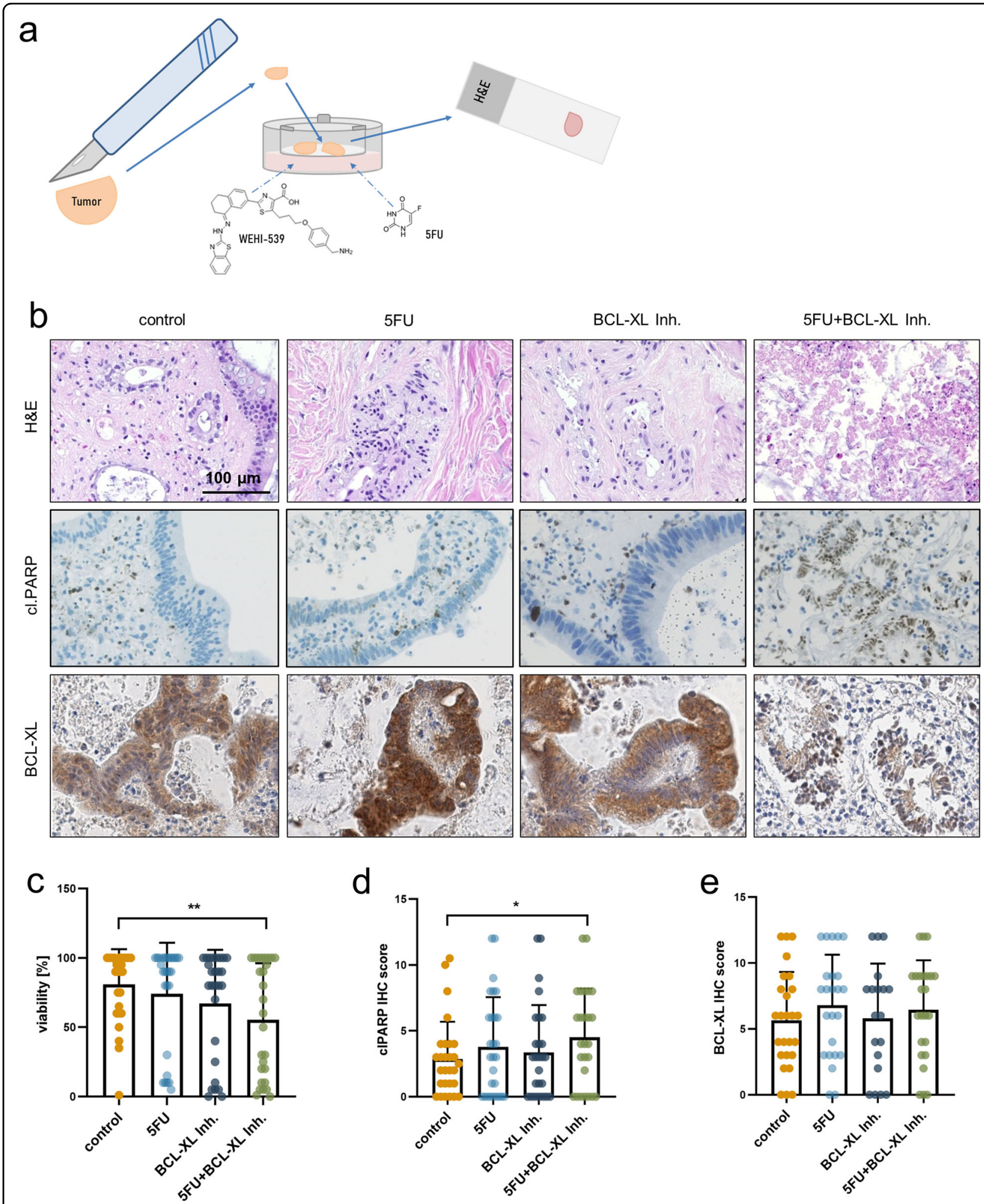

Fig. 6 Selective BCL-XL inhibition significantly enhances the antineoplastic effect of 5FU in patient-derived CRC specimens. a Schematic display of ex vivo cultures of human CRC specimens. Tumor samples were taken upon surgical resection, cut and kept in inserts at the air-liquid interface for $48 \mathrm{~h}$. Culture media were supplemented with $5 \mu \mathrm{M}$ WEHI-539, $50 \mu \mathrm{g} / \mathrm{ml} 5 \mathrm{FU}$ or the respective vehicle controls. b Representative H\&E staining (upper panel) and IHC staining against cleaved PARP (CI.PARP) and BCL-XL of patient-derived CRC specimens after treatment as depicted in $\mathbf{a}$. Scale bar indicates $100 \mu \mathrm{m}$. c Quantification of tumor cell viability based on the H\&E staining in $\mathbf{b}$. d Scoring of the IHC staining of clPARP depicted in $\mathbf{b}$. e Scoring of the $\mathrm{IHC}$ staining of $\mathrm{BCL}-\mathrm{XL}$ depicted in $\mathbf{b}$. Results in $\mathbf{c}-\mathbf{e}$ are shown as individual values with boxes depicting mean values; ${ }^{*} p<0.05 ;{ }^{* *} p<0.01$. 
lines ${ }^{45}$. Since increased expression of antiapoptotic BCL-2 proteins induces therapy resistance in a variety of human tumors ${ }^{49-51}$, we sought to evaluate whether basal BCL-XL levels might influence the response to the standard-ofcare chemotherapeutic agents 5FU and irinotecan. For both drugs, we found a similar pattern and an inverse correlation of BCL-XL expression and cell death in response to chemotherapeutic treatment. Taken together, these findings point to a potential role of $\mathrm{BCL}-\mathrm{XL}$ in chemotherapy resistance.

In order to evaluate the potential of a combined approach, we treated human CRC cells with WEHI-539, $5 \mathrm{FU}$ or irinotecan or combinations thereof. In all constellations, we found a significantly enhanced cell death induction under combined treatment. Lately, a direct binding of irinotecan to BCL-XL has been described as an additional mode of action besides its known function as DNA topoisomerase I inhibitor ${ }^{52}$. This might explain why the combination of WEHI-539 with irinotecan is even more efficient than the combination with 5FU. However, because $5 \mathrm{FU}$ is part of both standard regimens for the treatment of CRC (FOLFOX and FOLFIRI), we chose this therapeutic agent for further in vivo experiments. By utilizing a well-established chemically induced CRC mouse model ${ }^{40}$, we were able to show that the combination of 5FU and A-1331852, which is an orally available derivative of WEHI- $539^{35}$, but not the respective monotherapies, significantly decreased tumor size and overall tumor burden. This is in-line with a previous study by Leverson et al. in which A-1331852 was applied as single agent or in combination with docetaxel. In several subcutaneous xenograft models the authors found only modest anti-tumor activity of the BCL-XL inhibitor alone, which was however strongly enhanced in the presence of docetaxel ${ }^{35}$.

In contrast to tumor cells, neither the murine intestinal mucosa nor human intestinal epithelial cells were affected in their viability by BCL-XL inhibition. In mice, the good treatment tolerability was furthermore mirrored by the unchanged average body weight in the treated groups. Taken together, our data indicate that targeting BCL-XL has cancer cell specific effects while sparing the healthy epithelial cells.

The first clinical trials with the dual BCL-2/BCL-XL inhibitor ABT-263 revealed that the dose-limiting side effects are leukopenia and thrombocytopenia ${ }^{53,54}$. Whereas leukopenia was mainly attributed to the inhibition of BCL-2, inhibition of BCL-XL is presumably the major cause of thrombocytopenia observed upon ABT263 treatment $^{35}$. In-line with this, we found no significant difference in the number of leukocytes in BCL-XL inhibitor-treated mice. Although the number of thrombocytes was significantly reduced by the BCL-XL inhibitor, we found no spontaneous bleeding in the treated mice.
Besides thrombocytopenia, the hemogram revealed lowered erythrocyte and hemoglobin levels after inhibitor treatment. Reduced hemoglobin levels could be caused by an apoptosis-independent role of BCL-XL, as shown in a previous study postulating a requirement of BCL-XL for heme synthesi ${ }^{55}$. Furthermore, BCL-XL seems to prevent cell death induction in late-stage erythroblasts ${ }^{56}$. Nevertheless, the adverse effects on platelet counts and hemoglobin levels seem manageable by appropriate dosing and timing of inhibitor treatment. In sum, our in vivo studies suggest that specific targeting of BCL-XL has moderate side effects on the hematopoietic system.

Translating the findings from the mouse model in a closer-to-patient setting, we treated 27 patient-derived CRC specimens with WEHI-539, 5FU or the combination thereof ex vivo. The results support our in vivo findings, because a significant decrease of tumor cell viability was only observed under combinatorial but not mono-drug treatment. Taken together, this study identifies BCL-XL among the group of antiapoptotic BCL-2 proteins as the most promising target for CRC treatment. Furthermore, we proved in vitro, in vivo and ex vivo that additional inhibition of BCL-XL augments the efficacy of 5FU. This raises the possibility to render tumor cells susceptible towards lower doses of chemotherapy or even overcome treatment resistance. In addition, we could show in the murine model that nontransformed intestinal epithelial cells stay unaffected by the inhibition of BCL-XL and that treatment-efficient inhibitor doses have a good tolerability without unmanageable adverse effects. These findings might pave the way for future clinical applications.

\section{Acknowledgements}

We thank Ulrike Gärtner, Klaus Hillesheim and Silvio Krasemann from the Interfaculty Biomedical Research Facility (IBF) of the University Heidelberg, Germany, who helped by conducting the animal experiments. Whole-genome and RNA sequencing were performed within the MASTER program supported by the Molecular Diagnostics Program of NCT Heidelberg and the German Cancer Consortium. We thank the NCT/DKFZ Sample Processing Laboratory, the DKFZ Genomics and Proteomics Core Facility, and the DKFZ Omics IT and Data Management Core Facility for technical support. Furthermore, we thank Marion Hoffer for helping with the sample collection. Open Access funding enabled and organized by Projekt DEAL.

\section{Author details}

'Department of Medical Oncology, National Center for Tumor Diseases (NCT), University Hospital Heidelberg, 69120 Heidelberg, Germany. ${ }^{2}$ Department of Translational Medical Oncology, National Center for Tumor Diseases (NCT) Heidelberg, German Cancer Research Center (DKFZ), 69120 Heidelberg, Germany. ${ }^{3}$ German Cancer Consortium (DKTK), 69120 Heidelberg, Germany. ${ }^{4}$ Institute of Pathology, University Hospital Heidelberg, 69120 Heidelberg, Germany. ${ }^{5}$ Department of Medicine V, University Hospital Heidelberg, 69120 Heidelberg, Germany. ${ }^{6}$ Department of General, Visceral and Transplantation Surgery, University of Heidelberg, 69120 Heidelberg, Germany. ${ }^{7}$ Department of Medicine III, University Hospital, LMU Munich, 81377 Munich, Germany. ${ }^{8}$ Department of Molecular Medicine, Interfaculty Institute for Biochemistry, University of Tübingen, 72076 Tübingen, Germany. ${ }^{9}$ Department of Internal Medicine I, Medical Center-University of Freiburg, Faculty of Medicine, University of Freiburg, 79106 Freiburg, Germany. ${ }^{10}$ German Cancer Consortium (DKTK) Partner Site Freiburg and German Cancer Research Center (DKFZ), 69120 Heidelberg, Germany. ${ }^{11}$ Comprehensive Cancer Center Freiburg (CCCF), 
Medical Center-University of Freiburg, Faculty of Medicine, University of Freiburg, 79106 Freiburg, Germany. ${ }^{12}$ Institute of Medical Bioinformatics and Systems Medicine, Medical Center-University of Freiburg, Faculty of Medicine, University of Freiburg, 79106 Freiburg, Germany. ${ }^{13}$ Department of Medicine 1, University Hospital Frankfurt, 60590 Frankfurt, Germany. ${ }^{14}$ Universitäres Centrum für Tumorerkrankungen (UCT), University Hospital Frankfurt, 60590 Frankfurt, Germany. ${ }^{15}$ Depārtment of Medical Oncology, Sarcoma Center, West German Cancer Center, University Duisburg-Essen, Medical School, 45147 Essen, Germany. ${ }^{16}$ DKTK partner site Essen and German Cancer Consortium (DKTK), 69120 Heidelberg, Germany. ${ }^{17}$ Institute for Developmental Cancer Therapeutics, West German Cancer Center, University Hospital Essen, 45147 Essen, Germany. ${ }^{18}$ Division of Solid Tumor Translational Oncology, German Cancer Consortium (DKTK, partner site Essen) and German Cancer Research Center, DKFZ, 69120 Heidelberg, Germany. ${ }^{19}$ Medical Department III for Hematology and Oncology, School of Medicine, Klinikum rechts der Isar, Technical University of Munich, 81675 Munich, Germany. ${ }^{20}$ Central Institute for Translational Cancer Research (Translatum), Technical University of Munich, 81675 Munich, Germany. ${ }^{21}$ German Consortium for Translational Cancer Research (DKTK) partner site TUM, German Cancer Research Center Heidelberg (DKFZ), 69120 Heidelberg, Germany. ${ }^{22}$ Department of Internal Medicine I, University Hospital Tübingen, 72076 Tübingen, Germany. ${ }^{23}$ Charité Comprehensive Cancer Center, 10117 Berlin, Germany. ${ }^{24}$ Department of Hematology, Oncology and Tumor Immunology (CCM) Charité Universitaetsmedizin Berlin, 10117 Berlin, Germany. ${ }^{25}$ Division of Applied Bioinformatics, German Cancer Research Center (DKFZ), 69120 Heidelberg, Germany. ${ }^{26}$ Department of Translational Medical Oncology, National Center for Tumor Diseases (NCT) Dresden and German Cancer Research Center (DKFZ), 01307 Dresden, Germany. ${ }^{27}$ Center for Personalized Oncology, University Hospital Carl Gustav Carus Dresden at TU Dresden, 01307 Dresden, Germany. ${ }^{28}$ German Cancer Consortium (DKTK) Dresden, 01307 Dresden, Germany. ${ }^{29}$ Department of General and Visceral Surgery, Spital Linth, 8730 Uznach, Switzerland. ${ }^{30}$ Department of Internal Medicine II, Marien-Hospital, 46483 Wesel, Germany. ${ }^{31}$ Division of Chronic Inflammation and Cancer, German Cancer Research Center (DKFZ), 69120 Heidelberg, Germany

\section{Author contributions}

B.C.K., A.L.S., and S.F. designed the experiments, which were performed by A.L. S., N.S., A.M., and P.R. Data analysis and statistics were done by A.M., C.E.H., G.G., A.L.S., P.H., and F.K. Samples were collected by B.B., H.G., A.S., M.H., S.G., T.G., K.H.M., K.S.O., A.L.I., M.B., J.T., O.W., J.F., J.S., L.V., I.J., P.J.J., M.I.B., N.P.M., and M.S. D.J., H.S.B., B.G., P.S., and B.C.K. supervised the project. The manuscript was written by A.L.S., B.C.K., S.F., and A.M. and revised by all other co-authors. All authors read and approved the final version of the manuscript.

\section{Conflict of interest}

The authors declare that they have no conflict of interest.

\section{Publisher's note}

Springer Nature remains neutral with regard to jurisdictional claims in published maps and institutional affiliations.

Supplementary Information accompanies this paper at (https://doi.org/ 10.1038/s41419-020-03092-7).

Received: 10 June 2020 Revised: 1 October 2020 Accepted: 2 October 2020 Published online: 17 October 2020

\section{References}

1. Brenner, H., Kloor, M. \& Pox, C. P. Colorectal cancer. Lancet Lond. Engl. 383 1490-1502 (2014)

2. Keum, N. \& Giovannucci, E. Global burden of colorectal cancer: emerging trends, risk factors and prevention strategies. Nat. Rev. Gastroenterol. Hepatol. https://doi.org/10.1038/s41575-019-0189-8 (2019).

3. Kuipers, E. J. et al. Colorectal cancer. Nat. Rev. Dis. Prim. 1, 15065 (2015).

4. Tol, J. et al. Chemotherapy, bevacizumab, and cetuximab in metastatic colorectal cancer. N. Engl. J. Med. 360, 563-572 (2009).
5. Menter, D. G. et al. Back to the colorectal cancer consensus molecular subtype future. Curr. Gastroenterol. Rep. 21, 5 (2019).

6. Van Cutsem, E. et al. Binimetinib, encorafenib, and cetuximab triplet therapy for patients with BRAF V600E-mutant metastatic colorectal cancer: safety leadin results from the phase III BEACON colorectal cancer study. J. Clin. Oncol. 37, 1460-1469 (2019).

7. Guler, I., Askan, G., Klostergaard, J. \& Sahin, I. H. Precision medicine for metastatic colorectal cancer: an evolving era. Expert Rev. Gastroenterol. Hepatol. https://doi.org/10.1080/17474124.2019.1663174 (2019).

8. Morse, M. A., Hochster, H. \& Benson, A. Perspectives on treatment of metastatic colorectal cancer with immune checkpoint inhibitor therapy. Oncologist https://doi.org/10.1634/theoncologist.2019-0176 (2019).

9. Koehler, B. C., Jäger, D. \& Schulze-Bergkamen, H. Targeting cell death signaling in colorectal cancer: current strategies and future perspectives. World J. Gastroenterol. 20, 1923-1934 (2014).

10. Hu, T., Li, Z., Gao, C.-Y. \& Cho, C. H. Mechanisms of drug resistance in colon cancer and its therapeutic strategies. World J. Gastroenterol. 22, 6876-6889 (2016).

11. Burgess, D. J. Apoptosis: refined and lethal. Nat. Rev. Cancer 13, 79 (2013).

12. Certo, M. et al. Mitochondria primed by death signals determine cellular addiction to antiapoptotic BCL-2 family members. Cancer Cell 9, 351-365 (2006).

13. Chonghaile, T. N. et al. Pretreatment mitochondrial priming correlates with clinical response to cytotoxic chemotherapy. Science 334, 1129-1133 (2011).

14. Bender, A. et al. PI3K inhibitors prime neuroblastoma cells for chemotherapy by shifting the balance towards pro-apoptotic $\mathrm{BCl}-2$ proteins and enhanced mitochondrial apoptosis. Oncogene 30, 494-503 (2011).

15. Faber, A. C. et al. Differential induction of apoptosis in HER2 and EGFR addicted cancers following PI3K inhibition. Proc. Natl Acad. Sci. USA 106, 19503-19508 (2009).

16. Deng, J. et al. Proapoptotic $\mathrm{BH} 3-$ only $\mathrm{BCL}-2$ family protein BIM connects death signaling from epidermal growth factor receptor inhibition to the mitochondrion. Cancer Res. 67, 11867-11875 (2007).

17. Czabotar, P. E., Lessene, G., Strasser, A. \& Adams, J. M. Control of apoptosis by the BCL-2 protein family: implications for physiology and therapy. Nat. Rev. Mol. Cell Biol. 15, 49-63 (2014).

18. Youle, R. J. \& Strasser, A. The BCL-2 protein family: opposing activities that mediate cell death. Nat. Rev. Mol. Cell Biol. 9, 47-59 (2008).

19. Green, D. R. \& Walczak, H. Apoptosis therapy: driving cancers down the road to ruin. Nat. Med. 19, 131-133 (2013).

20. Deeks, E. D. Venetoclax: first global approval. Drugs 76, 979-987 (2016).

21. Cang, S., Iragavarapu, C., Savooji, J., Song, Y. \& Liu, D. ABT-199 (venetoclax) and BCL-2 inhibitors in clinical development. J. Hematol. Oncol. J. Hematol. Oncol. 8, 129 (2015).

22. Green, D. R. A BH3 mimetic for killing cancer. Cells Cell 165, 1560 (2016).

23. Knight, T., Edwards, H., Taub, J. W. \& Ge, Y. Evaluating venetoclax and its potential in treatment-naïve acute myeloid leukemia. Cancer Manag. Res. 11 3197-3213 (2019)

24. Beroukhim, R. et al. The landscape of somatic copy-number alteration across human cancers. Nature 463, 899-905 (2010).

25. Scherr, A.-L. et al. BCl-xL is an oncogenic driver in colorectal cancer. Cell Death Dis. 7, e2342 (2016)

26. Cho, S.-Y. et al. A novel combination treatment targeting $B C L-X L$ and MCL1 for KRAS/BRAF-mutated and BCL2L1-amplified colorectal cancers. Mol. Cancer Ther. 16, 2178-2190 (2017)

27. Horak, P. et al. Precision oncology based on omics data: the NCT Heidelberg experience. Int. J. Cancer 141, 877-886 (2017).

28. Califano, A. \& Alvarez, M. J. The recurrent architecture of tumour initiation, progression and drug sensitivity. Nat. Rev. Cancer 17, 116-130 (2017).

29. Ding, $H$. et al. Quantitative assessment of protein activity in orphan tissues and single cells using the metaVIPER algorithm. Nat. Commun. 9, 1-10 (2018).

30. Alvarez, M. J. Virtual inference of protein-activity by enriched regulon analysis. (Bioconductor version: Release (3.11)). https://doi.org/10.18129/B9.bioc.viper (2020).

31. Basso, K. et al. Reverse engineering of regulatory networks in human B cells. Nat. Genet. 37, 382-390 (2005).

32. Giorgi, F. M. ARACNe-inferred gene networks from TCGA tumor datasets. http:// bioconductor.org/packages/aracne.networks/ (2020).

33. Koehler, B. C. et al. Beyond cell death-antiapoptotic BCl-2 proteins regulate migration and invasion of colorectal cancer cells in vitro. PIOS ONE 8, e76446 (2013). 
34. Nicoletti, I., Migliorati, G., Pagliacci, M. C., Grignani, F. \& Riccardi, C. A rapid and simple method for measuring thymocyte apoptosis by propidium iodide staining and flow cytometry. J. Immunol. Methods 139, 271-279 (1991).

35. Leverson, J. D. et al. Exploiting selective BCL-2 family inhibitors to dissect cell survival dependencies and define improved strategies for cancer therapy. Sci. Transl. Med. 7, 279ra40 (2015).

36. Gdynia, G. et al. The HMGB1 protein induces a metabolic type of tumour cell death by blocking aerobic respiration. Nat. Commun. 7, 1-13 (2016).

37. Fasbender, F. \& Watzl, C. Impedance-based analysis of Natural Killer cell stimulation. Sci. Rep. 8, 4938 (2018)

38. Ferreira, K. S. et al. Caspase-3 feeds back on caspase-8, Bid and XIAP in type I Fas signaling in primary mouse hepatocytes. Apoptosis Int. J. Program. Cell Death 17, 503-515 (2012).

39. McComb, S. et al. Efficient apoptosis requires feedback amplification of upstream apoptotic signals by effector caspase-3 or -7. Sci. Adv. 5, eaau9433 (2019).

40. Neufert, C., Becker, C. \& Neurath, M. F. An inducible mouse model of colon carcinogenesis for the analysis of sporadic and inflammation-driven tumor progression. Nat. Protoc. 2, 1998-2004 (2007).

41. Reed, J. C. BCl-2-family proteins and hematologic malignancies: history and future prospects. Blood 111, 3322-3330 (2008).

42. Healy, M. E. et al. MCL1 is required for maintenance of intestinal homeostasis and prevention of carcinogenesis in mice. Gastroenterology 159, 183-199 (2020).

43. Placzek, W. J. et al. A survey of the anti-apoptotic BCl-2 subfamily expression in cancer types provides a platform to predict the efficacy of Bcl-2 antagonists in cancer therapy. Cell Death Dis. 1, e40 (2010).

44. Kønig, S. M., Rissler, V., Terkelsen, T., Lambrughi, M. \& Papaleo, E. Alterations of the interactome of $\mathrm{BCl}-2$ proteins in breast cancer at the transcriptional, mutational and structural level. PLoS Comput. Biol. 15, e1007485 (2019).
45. Zhang, $\mathrm{H}$. et al. Genomic analysis and selective small molecule inhibition identifies $B C L-X(L)$ as a critical survival factor in a subset of colorectal cancer. Mol. Cancer 14, 126 (2015).

46. Campbell, K. J. \& Tait, S. W. G. Targeting BCL-2 regulated apoptosis in cancer. Open Biol. 8, 180002 (2018).

47. Kotschy, A. et al. The MCL1 inhibitor S63845 is tolerable and effective in diverse cancer models. Nature 538, 477-482 (2016).

48. Lessene, G. et al. Structure-guided design of a selective $B C L-X(L)$ inhibitor. Nat. Chem. Biol. 9, 390-397 (2013).

49. Lee, E. F. et al. BCL-XL and $M C L-1$ are the key $B C L-2$ family proteins in melanoma cell survival. Cell Death Dis. 10, 1-14 (2019).

50. Yang, $Y$. et al. Reversing platinum resistance in ovarian cancer multicellular spheroids by targeting Bcl-2. OncoTargets Ther. 12, 897-906 (2019).

51. García-Aranda, M., Pérez-Ruiz, E. \& Redondo, M. Bcl-2 inhibition to overcome resistance to chemo- and immunotherapy. Int. J. Mol. Sci. 19, 3950 (2018).

52. Lee, B. et al. A novel mechanism of irinotecan targeting MDM2 and BCl-xL. Biochem. Biophys. Res. Commun. 514, 518-523 (2019).

53. Wilson, W. H. et al. Navitoclax, a targeted high-affinity inhibitor of BCL-2, in lymphoid malignancies: a phase 1 dose-escalation study of safety, pharmacokinetics, pharmacodynamics, and antitumour activity. Lancet Oncol. 11, 1149-1159 (2010).

54. Puglisi, M. et al. A phase I safety and pharmacokinetic (PK) study of navitoclax $(\mathrm{N})$ in combination with docetaxel (D) in patients (pts) with solid tumors. J. Clin. Oncol. 29, 2518-2518 (2011).

55. Hafid-Medheb, K., Augery-Bourget, Y., Minatchy, M.-N., Hanania, N. \& RobertLézénès, J. BCl-XL is required for heme synthesis during the chemical induction of erythroid differentiation of murine erythroleukemia cells independently of its antiapoptotic function. Blood 101, 2575-2583 (2003).

56. Rhodes, M. M., Kopsombut, P., Bondurant, M. C., Price, J. O. \& Koury, M. J. BCl-xL prevents apoptosis of late-stage erythroblasts but does not mediate the antiapoptotic effect of erythropoietin. Blood 106, 1857-1863 (2005). 